\title{
Extended Major Histocompatibility Complex Haplotypes in Patients with Gluten-sensitive Enteropathy
}

\author{
Chester A. Alper, Ellen Fleischnick, Zuheir Awdeh, Aubrey J. Katz, and Edmond J. Yunis \\ The Center for Blood Research, Departments of Pediatrics and Pathology, Harvard Medical School, \\ Dana-Farber Cancer Institute, and The Children's Hospital, Boston, Massachusetts 02115
}

\begin{abstract}
We have studied major histocompatibility complex markers in randomly ascertained Caucasian patients with gluten-sensitive enteropathy and their families. The frequencies of extended haplotypes, defined as haplotypes of specific HLA-B, DR, BF, C2, C4A, and C4B allelic combinations, occurring more frequently than expected, were compared on patient chromosomes, on normal chromosomes from the study families, and on chromosomes from normal families. Over half of patient chromosomes consisted almost entirely of two extended haplotypes [HLA-B8, DR3, SC01] and [HLA-B44, DR7, FC31] which, with nonextended HLA-DR7, accounted for the previously observed HLA markers of this disease: HLA-B8, DR3, and DR7. There was no increase in HLA-DR3 on nonextended haplotypes or in other extended haplotypes with HLA-DR3 or DR7. The distribution of homozygotes and heterozygotes for HLA-DR3 and DR7 was consistent with recessive inheritance of the major histocompatibility complex-linked susceptibility gene for gluten-sensitive enteropathy. On the other hand, by odds ratio analysis and from the sum of DR3 and DR7 homozygotes compared with DR3/ DR7 heterozygotes, there was an increase in heterozygotes and a decrease in homozygotes suggesting the presence of modifying phenomena.
\end{abstract}

\section{Introduction}

Gluten-sensitive enteropathy or celiac disease is a malabsorption syndrome caused by an abnormal sensitivity to wheat and rye gluten. Patients with the disease have severe blunting or absence of villi in the small intestine on biopsy. There is a complete reversion to a normal villus pattern and to normal absorption of food after the removal of gluten from the diet. Although symptoms usually begin in early childhood, the onset may be delayed until adulthood.

Caucasian patients with gluten-sensitive enteropathy have increased frequencies of HLA-A1, B8, Dw3/DR3, and Dw7/ DR7 and a decreased frequency of HLA-B7 compared with normal controls (1-4). Because the relative risk of HLA-A1 is lower than that of HLA-B8, the latter has been considered primary.

There are four complement genes in the major histocompatibility complex (MHC) between HLA-B and DR. They are inherited as single genetic units called "complotypes" and designated by their BF, C2, C4A, and C4B alleles. Some specific complotype, HLA-B, DR allelic sets occur on random Caucasian

Received for publication 21 March 1986 and in revised form 7 July 1986.

J. Clin. Invest.

(c) The American Society for Clinical Investigation, Inc.

0021-9738/87/01/0251/06 \$1.00

Volume 79, January 1987, 251-256 chromosomes in significant positive-linkage disequilibrium (their frequency as a haplotype significantly exceeds the frequency predicted by the frequencies of the alleles of which they are composed). These are called "extended haplotypes."

Like gluten-sensitive enteropathy, type I diabetes mellitus is an MHC-linked and MHC allele-associated disease. In patients with type I diabetes mellitus, there are increased frequencies of HLA-B8, B18, Bw62, DR3, DR4, and the rare complement alleles $B F^{*} F 1$ and $C 4 B^{*} 3(5-10)$. The increased frequencies of these individual alleles are by and large the result of increased frequencies in a few extended haplotypes which carry them: [HLA-B8, DR3, SC01], [HLA-B18, DR3, F1C30], and [HLABw62, DR4, SC33] (11). In diabetes, [HLA-B8, DR3, SC01] in patients, in most instances, carries HLA-A1 (as does this haplotype in normal individuals) and the allele GLO2 rather than a roughly equal proportion of GLO1 and GLO2 as on normal haplotypes.

The present study is of MHC haplotypes in patients with gluten-sensitive enteropathy and their families. Over half of haplotypes from randomly selected, unrelated Caucasian patients with gluten-sensitive enteropathy were found to be accounted for by two extended haplotypes, which in turn account for nearly all the reported positive MHC associations with gluten-sensitive enteropathy.

\section{Methods}

Blood samples from 24 random unrelated Caucasian patients with glutensensitive enteropathy and their families were collected into 10-ml Vacutainer tubes (Becton, Dickinson \& Co., Rutherford, NJ) containing 10.5 $\mathrm{mg}$ of potassium EDTA and into a heparinized syringe containing 500$1,000 \mathrm{U}$ of sodium heparin and diluted with an equal volume of RPMI 1640. Lymphocytes were separated by Ficoll-Hypaque, frozen, and stored in vapor-phase liquid nitrogen until thawed for analysis. Plasma was separated by centrifugation and stored at $-80^{\circ} \mathrm{C}$ and thawed just before analysis. No family had more than one affected patient. The diagnosis was established by history, by small intestinal biopsy on gluten-containing diet, and in most instances, by repeat biopsies on and off a gluten-containing diet. The findings of flat villi on gluten and normalization on a gluten-free diet were considered diagnostic.

For the study of C4 polymorphism, plasma samples were incubated with neuraminidase from Clostridium perfringens (type VI, Sigma Chemical Co., St. Louis, MO) at a concentration of $10 \mathrm{mU} / \mu \mathrm{l}$ of plasma for $15 \mathrm{~h}$ at $4^{\circ} \mathrm{C}$ with continuous dialysis against $0.1 \mathrm{M}$ phosphate, $\mathrm{pH}$ 6.8 , containing $0.005 \mathrm{M}$ sodium EDTA. These desialated samples were then subjected to electrophoresis in $0.75 \%$ agarose gel using a Tris/glycine/ barbital discontinuous buffer system and to immunofixation with antihuman C4 (Atlantic Antibodies, Scarborough, ME) as described earlier (12). In some cases, samples were also processed as for immunofixation, but were developed with a fourth component of complement (C4) functional overlay consisting of $1 \%$ antibody-sensitized sheep erythrocytes in $0.6 \%$ isotonic barbital-buffered saline containing $\mathrm{Ca}^{2+}, \mathrm{Mg}^{2+}$, and hydrazine-treated normal human serum or C4-deficient guinea pig serum. The presence of null alleles was determined by visual inspection of immunofixation patterns or by crossed immunoelectrophoresis (13). 
The nomenclature used for $\mathrm{C} 4$ is that described previously $(12,14)$. Variants and alleles at each locus have been designated with integers according to the electrophoretic mobility of the desialated proteins at $\mathrm{pH} 8.8$ from cathode to anode. Individual alleles are italicized and designated by locus name in capital letters, an asterisk, and a number or the symbol $Q 0$ if null (e.g., $C 4 A^{*} 2, C 4 B^{*} 1$, or $C 4 B^{*} Q 0$ ). Phenotypes, variants, or proteins are designated with roman capital letters, a space, and the same number or symbol as the corresponding allele.

For BF typing, plasma samples were subjected to agarose gel electrophoresis and immunofixation with anti-human factor B (15) (Atlantic Antibodies). For second component of complement (C2) typing, samples were subjected to isoelectric focusing in thin-layer polyacrylamide gel (16), using human serum at 1:90 instead of $\mathrm{C} 2$-deficient serum in the overlay to develop $\mathrm{C} 2$ patterns.

Complotypes are designated by their BF, C2, C4A, and C4B alleles, in that (arbitrary) order (17). Null or Q0 alleles are simply 0 . Thus, $\mathrm{SCO} 1$ stands for $B F^{*} S, C 2^{*} C, C 4 A^{*} Q 0, C 4 B^{*} l$. The $48 \mathrm{MHC}$ haplotypes occurring in the 24-patient propositi constituted the gluten-sensitive enteropathy disease chromosomes. A group of $714 \mathrm{MHC}$ haplotypes from random Caucasian patients without MHC-related disease themselves or in close blood relatives was used to construct a population of 357 "normalnormal individuals" assuming the Hardy-Weinberg equilibrium. An additional and separate control population was comprised of the 59 chromosomes from the study families that did not occur in patients (11). This population of normal chromosomes from the study families was used to assure ethnic matching for the disease chromosomes and is referred to as "family-normal."

HLA-A, B, and C antigens were identified by the standard National Institutes of Health lymphocyte microcytotoxicity assay using 140 antisera to define 19 A-locus, 28 B-locus, and 6 C-locus antigens. HLA-DR typing was done with 70 well-characterized alloantisera using the technique of the Oxford (Seventh International Histocompatibility) Workshop (18).

Glyoxalase I (GLO) ${ }^{1}$ types were determined by electrophoresis of red cell lysates on cellulose acetate membranes in a $0.03 \mathrm{M}$ Tris, $0.03 \mathrm{M}$ barbituric acid, $0.2 \mathrm{mM}$ 2-mercaptoethanol, $0.4 \mathrm{mM} \mathrm{MgCl} 2$ buffer at pH 8.0 (19). Electrophoresis was carried out at $200 \mathrm{~V}$ for $1 \mathrm{~h}$ or until a human serum albumin marker had migrated $10 \mathrm{~cm}$. Membranes were stained first with $0.02 \mathrm{M}$ glutathione and $0.34 \mathrm{M}$ methylglyoxal in a 0.1 $\mathrm{M}$ phosphate buffer at $\mathrm{pH} 6.5$ and then with $0.1 \mathrm{M}$ 3-[4,5-dimethylthiazolyl-2]-2,5-diphenyltetrazolium bromide, and $0.69 \mathrm{M}$ 2,4-dichlorophenylindophenol in a $0.1 \mathrm{M}$ Tris hydrochloride buffer at $\mathrm{pH} 7.8$.

Statistical significance was determined by $\chi^{2}$ analysis using either (observed - expected) ${ }^{2} /$ expected or $2 \times 2$ contingency tables.

\section{Results}

Almost all (95.8\%) of patients with gluten-sensitive enteropathy were positive for HLA-DR3, DR7, or both. Of the 48 patient chromosomes, $29 \%$ or $60 \%$ carried extended haplotypes compared with 176 of 714 normal chromosomes or $25 \%\left(\chi^{2}=29.26\right.$; $P<0.001$ ) (Tables I and II). Of the patient extended haplotypes, there were single instances of [HLA-B7, DR2, SC31] and [HLA$B 18, D R 3, F 1 C 30]$, and the remaining extended haplotypes were either [HLA-B8, DR3, SC01] or [HLA-B44, DR7, FC31].

The distribution of MHC haplotypes by the HLA-DR allele they carry is shown in Fig. 1 for patient chromosomes, ethnically matched family-normal chromosomes and normal-normal chromosomes. Each bar in Fig. 1 is segmented to show the absolute contribution of extended haplotypes for each kind of chromosome. The well-known increases in HLA-DR3 and HLADR7 in patients are clearly seen. It is further obvious that the

1. Abbreviation used in this paper: GLO, glyoxalase I. increases in these DR types are exclusively or largely the result of increases in two extended haplotypes; [HLA-B8, DR3, SC01] (carrying either GLO1 or GLO2) and [HLA-B44, DR7, FC31]. Other extended haplotypes with HLA-DR3 such as [HLA-B18, DR3, F1C30] or with HLA-DR7 such as [HLA-Bw57, DR7, SC61] are not increased on patient haplotypes. It is also clear that haplotypes with other HLA-DR alleles and other extended haplotypes tend to be reduced among patient chromosomes. Fig. 1 also demonstrates that HLA-DR3 not on extended haplotypes is not increased on patient chromosomes. On the other hand, there was an increase in HLA-DR7 not on extended haplotypes.

Table II gives statistical analyses of the distribution of extended haplotypes among patient, family-normal, and normalnormal chromosomes. The increases in [HLA-B44, DR7, FC31] and [HLA-B8, DR3, SC01] on patient compared with either of the control chromosome populations are highly significant $(P$ $<0.001$ ). Although not shown, the frequencies of both the GLO1 and GLO2 forms of [HLA-B8, DR3, SC01] are highly significantly increased over their normal frequencies $\left(\chi^{2}=15.17\right.$ and 48.51 , respectively, both $P<0.001$ for the comparison with normal-normal haplotypes). That these striking differences were not because of different ethnicity between the patient and normal haplotypes is seen from the absence of statistically significant differences in HLA-DR and extended haplotype frequencies between family normal and normal-normal chromosomes (Table II).

As is evident from Table I, the patients were of a great variety of ethnic backgrounds. These did not appear to differ materially from our general Caucasian population in Boston. Table III gives the relative risks of HLA-DR and selected other MHC alleles and extended haplotypes on patient haplotypes compared with normal-normal haplotypes. It is evident that the increased relative risks of MHC alleles in relation to gluten-sensitive enteropathy can largely be ascribed to increases in the two extended haplotypes [HLA-B8, DR3, SC01] and [HLA-B44, DR7, FC31] which themselves have relative risks of 12.9 and 13.4, similar to or greater than the relative risks of HLA-DR3 (15.9) and DR7 (3.7).

Although the distribution of GLO alleles on patient [HLAB8, DR3, SC01] was not different from that on normal chromosomes with the same markers, the distribution of HLA-A alleles was different. Whereas over 78\% of [HLA-B8, DR3, SC01] in normals carry HLA-A1, HLA-A 1 is carried by only $37 \%$ of similar patient haplotypes $\left(\chi^{2}=15.00 ; P<0.001\right)$. 4 of 8 [HLAB44, DR7, FC31] from patients carried HLA-A29 compared with 3 of 19 normal-normal examples $\left(\chi^{2}=5.96\right.$; not significant if corrected for the number of comparisons). The increased relative risk of HLA-A29 clearly stems from this extended haplotype.

A comparison was made between the observed distribution of HLA-DR3, DR7, DR blank, and all other HLA-DR types among patients compared with that predicted by the HardyWeinberg equilibrium. This is shown in Table IV. There are no significant differences between observed and expected frequencies although there are nonsignificant decreases in HLA-DR3 and DR7 homozygotes and a nonsignificant increase in HLADR3/7 heterozygotes. On the other hand, the odds ratio for heterozygotes at $\mathbf{2 3 6}$ was considerably greater than that of HLADR3 homozygotes at 57 and of HLA-DR7 homozygotes at 71. Similarly, the sum of HLA-DR3 and HLA-DR7 homozygotes was 5 , considerably less than the number of heterozygotes at 10 . 
Table I. Age of Onset, Ethnicity, and MHC Haplotypes of Patients with Gluten-sensitive Enteropathy

\begin{tabular}{|c|c|c|c|c|c|c|c|c|c|}
\hline Family no. & Parental origin & Ethnicity* & Age of onset & HLA-A & HLA-B & HLA-C & HLA-DR & Complotype & GLO \\
\hline \multirow[t]{2}{*}{825} & $\mathbf{P}$ & $\mathrm{E}$ & $5 \mathrm{yr}$ & 25 & 8 & & 3 & SC01 & 2 \\
\hline & $\mathbf{M}$ & $\mathrm{E}$ & & 26 & 45 & & 7 & FC01 & 2 \\
\hline \multirow[t]{2}{*}{916} & $\mathbf{P}$ & - & $2 \mathrm{yr}$ & 29 & 44 & & 7 & FC31 & $1 / 2$ \\
\hline & $\mathbf{M}$ & Ir, Swe & & 1 & 8 & & 3 & $\mathrm{SCO} 1$ & $1 / 2$ \\
\hline \multirow[t]{2}{*}{927} & $\mathbf{P}$ & $\mathbf{R}$ & $18 \mathrm{mo}$ & 30 & 13 & w6 & 7 & SC31 & 2 \\
\hline & $\mathbf{M}$ & Ir, Fr, E & & 32 & 8 & & 3 & $\mathrm{SC} 01$ & 2 \\
\hline \multirow[t]{2}{*}{930} & $\mathbf{P}$ & $\mathbf{A J}$ & $7 \mathrm{mo}$ & 1 & 8 & & 3 & $\mathrm{SCO} 1$ & 2 \\
\hline & $\mathbf{M}$ & $\mathrm{AJ}$ & & 30 & 38 & & 5 & SC31 & 2 \\
\hline \multirow[t]{2}{*}{974} & $\mathbf{P}$ & Ir & $8 \mathrm{yr}$ & 1 & 8 & & 3 & SCO1 & 1 \\
\hline & $\mathbf{M}$ & Ir & & 2 & 8 & & 3 & SCO1 & 2 \\
\hline \multirow[t]{2}{*}{986} & $\mathbf{P}$ & $\mathbf{A J}$ & $15 \mathrm{mo}$ & 29 & 14 & & 7 & SC31 & 1 \\
\hline & $\mathbf{M}$ & $\mathrm{AJ}$ & & 3 & 7 & & 5 & $\mathrm{SC} 31$ & 2 \\
\hline \multirow[t]{2}{*}{990} & $\mathbf{P}$ & Ir, Sc, E & $18 \mathrm{yr}$ & 1 & 8 & & 3 & $\mathrm{SC} 01$ & 1 \\
\hline & $\mathbf{M}$ & $\mathrm{E}, \mathrm{Sc}$ & & 2 & 44 & & 7 & FC31 & 1 \\
\hline \multirow[t]{2}{*}{991} & $\mathbf{P}$ & It & $17 \mathrm{mo}$ & 24 & 51 & & 5 & SC31 & $1 / 2$ \\
\hline & $\mathbf{M}$ & Fr, E & & 2 & 44 & & 7 & FC31 & $1 / 2$ \\
\hline \multirow[t]{2}{*}{994} & $\mathbf{P}$ & Ger & $18 \mathrm{mo}$ & 3 & 7 & & 2 & SC31 & $1 / 2$ \\
\hline & $\mathbf{M}$ & Ger & & 2 & 44 & & 5 & SC30 & $1 / 2$ \\
\hline \multirow[t]{2}{*}{1005} & $\mathbf{P}$ & Por & $18 \mathrm{mo}$ & 34 & 8 & & 3 & SCO1 & 1 \\
\hline & $\mathbf{M}$ & $\mathrm{E}, \mathrm{Fr}$ & & 28 & 44 & & 7 & FC31 & 1 \\
\hline \multirow{2}{*}{1008} & $\mathbf{P}$ & Ir & $11 \mathrm{mo}$ & 2 & w62 & & 4 & SB42 & 1 \\
\hline & $\mathbf{M}$ & $\mathrm{E}, \mathrm{AI}$ & & 1 & 8 & & 3 & SC01 & 1 \\
\hline \multirow[t]{2}{*}{1012} & $\mathbf{P}$ & It, FC & $18 \mathrm{mo}$ & 24 & 8 & & 3 & SC01 & 2 \\
\hline & $\mathbf{M}$ & $\mathrm{E}, \mathrm{Ir}, \mathrm{Sc}$ & & 3 & 8 & & 3 & $\mathrm{SCO} 1$ & 2 \\
\hline \multirow[t]{2}{*}{1020} & $\mathbf{P}$ & Ger & $4 \mathrm{yr}$ & 1 & w57 & & - & SC61 & $1 / 2$ \\
\hline & $\mathbf{M}$ & It, E, Fr & & 29 & 44 & & 7 & FC31 & $1 / 2$ \\
\hline \multirow[t]{2}{*}{1036} & $\mathbf{P}$ & E, Ir, Swe & $5 \mathrm{yr}$ & 1 & 8 & & 1 & SC31 & 2 \\
\hline & $\mathbf{M}$ & Ir, E, Fr & & 29 & 8 & & 3 & SC01 & 2 \\
\hline \multirow[t]{2}{*}{1115} & $\mathbf{P}$ & Ger, Aus & $15 \mathrm{mo}$ & 29 & 44 & & 7 & FC31 & 2 \\
\hline & $\mathbf{M}$ & Ir, Por & & 2 & 8 & & 3 & SC01 & 1 \\
\hline \multirow[t]{2}{*}{1118} & $\mathbf{P}$ & Ir, Sc, E, Swe & $8 \mathrm{yr}$ & 28 & 8 & & 3 & $\mathrm{SC} 01$ & 2 \\
\hline & $\mathbf{M}$ & $\mathrm{E}$ & & 29 & 44 & & 7 & FC31 & 2 \\
\hline \multirow[t]{2}{*}{1119} & $\mathbf{P}$ & $\mathbf{R}$ & $18 \mathrm{mo}$ & w33 & 44 & & 7 & SC 31 & 2 \\
\hline & $\mathbf{M}$ & $\mathbf{R}$ & & 2 & 8 & & 3 & FC31 & 2 \\
\hline \multirow[t]{2}{*}{1120} & $\mathbf{P}$ & It & $5 \mathrm{yr}$ & 2 & 13 & w6 & 7 & SC31 & $1 / 2$ \\
\hline & $\mathbf{M}$ & It & & 26 & 14 & & 5 & SC31 & $1 / 2$ \\
\hline \multirow[t]{2}{*}{1121} & $\mathbf{P}$ & Ir, E, Sc & $9 \mathrm{mo}$ & 2 & 18 & w5 & 3 & F1C30 & 2 \\
\hline & $\mathbf{M}$ & $\mathrm{Ir}, \mathrm{E}$ & & 29 & 44 & & w6 & SC61 & 2 \\
\hline \multirow[t]{2}{*}{1122} & $\mathbf{P}$ & Ir, E & $10 \mathrm{mo}$ & 1 & 8 & & 3 & SCO1 & 1 \\
\hline & $\mathbf{M}$ & Ir & & 1 & w57 & & 7 & SC61 & 1 \\
\hline 1124 & $\mathbf{P}$ & Por & $12 \mathrm{yr}$ & 24 & 8 & & 3 & $\mathrm{SCO1}$ & 2 \\
\hline & $\mathbf{M}$ & Fr & & 2 & w60 & & 4 & SB42 & 1 \\
\hline 1125 & $\mathbf{P}$ & Fr, Ir, Ger & $15 \mathrm{mo}$ & 11 & 39 & & 3 & $\mathrm{SC} 42$ & 2 \\
\hline & $\mathbf{M}$ & Ger, Pol & & 24 & 22 & w1 & 7 & SC31 & 1 \\
\hline 1133 & $\mathbf{P}$ & Por & $11 \mathrm{yr}$ & 28 & 8 & & 3 & s.01 & 1 \\
\hline & $\mathbf{M}$ & Por & & 2 & 44 & w5 & 7 & FC31 & 1 \\
\hline 1481 & $P$ & Ger, Sc & $18 \mathrm{mo}$ & 1 & 8 & & 3 & $\mathrm{SCO}$ & 2 \\
\hline & $\mathbf{M}$ & Ir, It & & 11 & 51 & & w6 & FC30 & 1 \\
\hline
\end{tabular}

* Abbreviations: AI, American Indian; AJ, Ashkenazi Jewish; Aus, Austrian; E, English; Fr, French; FC, French Canadian; Ger, German; Ir, Irish; It, Italian; Pol, Polish; Por, Portuguese; R, Russian; Sc, Scottish; Swe, Swedish; Swi, Swiss.

\section{Discussion}

Our observations provide evidence that all the previously noted increases in HLA-B8, Dw3/DR3, and a major part of the increase in Dw7/DR7 among Caucasian patients with gluten-sensitive enteropathy are secondary to increases in two specific extended haplotypes [HLA-B8, DR3, SC01] and [HLA-B44, DR7, FC31]. Remarkable is the fact that these two extended haplotypes account for $56 \%$ of patient chromosomes. Among normal chromosomes or chromosomes from patients with type I diabetes, about half that proportion (25-30\%) of MHC haplotypes are extended (20). In keeping with our concept that other alleles of 
Table II. Extended MHC Haplotypes in Patients with Gluten-sensitive Enteropathy

\begin{tabular}{|c|c|c|c|c|c|c|c|c|}
\hline \multirow[b]{2}{*}{ Extended haplotype } & \multirow{2}{*}{$\begin{array}{l}\text { Frequency in } \\
\text { normal-normal } \\
\text { chromosomes } \\
(n=714)\end{array}$} & \multicolumn{4}{|l|}{ Observed } & \multicolumn{3}{|l|}{ Expected } \\
\hline & & $\begin{array}{l}\text { Patient chromosomes } \\
(n=48)\end{array}$ & $\begin{array}{l}\text { Family normal } \\
\text { chromosomes } \\
(n=59)\end{array}$ & $x^{2}$ & $P$ & $\begin{array}{l}\text { From normal- } \\
\text { normal } \\
\text { chromosomes }\end{array}$ & $x^{2}$ & $P$ \\
\hline [HLA-B8, DR3, SC01] & 0.083 & $19(0.40)^{*}$ & $5(0.08)$ & 54.6 & $<0.001$ & 4.0 & 56.9 & $<0.001$ \\
\hline [HLA-B7, DR2, SC31] & 0.046 & $1(0.02)$ & $5(0.08)$ & 2.3 & NS & 2.2 & - & NS \\
\hline [HLA-B44, DR4, SC30] & 0.029 & 0 & 0 & - & NS & 1.4 & - & NS \\
\hline [HLA-Bw62, DR4, SC33] & 0.017 & 0 & 0 & - & NS & 0.8 & - & NS \\
\hline [HLA-B44, DR7, FC31] & 0.018 & $8(0.17)$ & $2(0.03)$ & 24.9 & $<0.001$ & 0.9 & 58.4 & $<0.001$ \\
\hline [HLA-Bw57, DR7, SC61] & 0.028 & 0 & $2(0.03)$ & - & NS & 1.3 & - & NS \\
\hline [HLA-B35, DR 1, FC(3,2)0] & 0.011 & 0 & 0 & - & NS & 0.5 & - & NS \\
\hline [HLA-B18, DR3, F1C30] & 0.004 & $1(0.02)$ & $1(0.02)$ & - & NS & 0.2 & - & NS \\
\hline
\end{tabular}

* Numbers in parentheses are decimal fractions of haplotypes in each category.

such haplotypes are relatively fixed $(20,21)$, it seems likely that the disease susceptibility genes for gluten-sensitive enteropathy on these haplotypes are the result of two distinct mutations, each characteristic of one extended haplotype or the other. This might provide some insight into the reported differences in the responses in vitro to gliadin of lymphocytes from GSE patients with and without HLA-B8 $(22,23)$ or other markers of the extended haplotype [HLA-B8, DR3, SC01], inasmuch as different genes controlling such responses may also be trapped on different extended haplotypes. Also consistent with the fixity of alleles on extended haplotypes is the paucity of other extended haplotypes, because, if a disease susceptibility allele is not on an extended haplotype, that haplotype is rarely found in a patient population. HLA-B7 has previously been shown to be reduced in patients with gluten-sensitive enteropathy. HLA-B7 is often found on the extended haplotype [HLA-B7, DR2, SC31] and that is decreased on patient chromosomes. This same phenomenon is most clearly seen in the absence of [HLA-B8, DR3, SC01] from

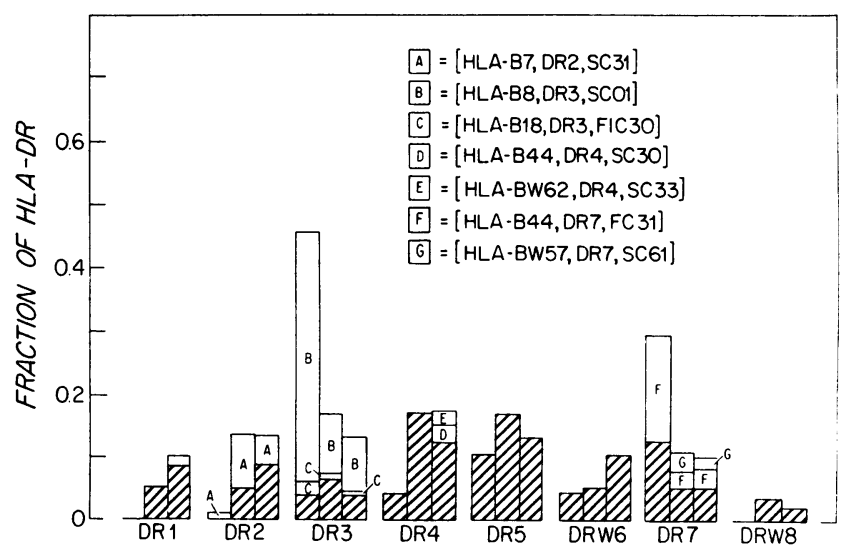

Figure 1. The frequencies of HLA-DR alleles are shown in a population of patients with gluten-sensitive enteropathy. For each HLA-DR allele the bar on the left is the frequency in patients with gluten-sensitive enteropathy; the bar in the center is the frequency of the normal, non-disease-associated haplotypes from the same families as the patient chromosomes. The bar on the right of each set is the frequency of these alleles in all normals. Within each bar, extended haplotypes are denoted by clear areas and are labeled as $A, B, C$, etc. These correspond to the haplotypes in the upper right-hand corner. the chromosomes of patients with 21-hydroxylase deficiency congenital adrenal hyperplasia (24). The gene for that disorder is known to be in the MHC (25-27).

HLA-DR 3 and DR4 are increased among patients with type I diabetes, but the ratio of DR3/DR4 heterozygotes is greater than expected from the Hardy-Weinberg equilibrium (assuming pure recessive inheritance) compared with homozygotes for either HLA-DR3 or DR4 $(28,29)$. In calculating the relative risks of these different classes of patients, it is important to use the same reference population (non-DR3, non-DR4) in a calculation yielding an odds ratio (28). An odds ratio for the heterozygote greater than the homozygotes was interpreted by Svejgaard and Ryder as being consistent with "overdominant" inheritance, although other explanations including multiple gene interactions are possible. Since gluten-sensitive enteropathy also has two

Table III. Relative Risks of MHC Markers in Patients with Gluten-sensitive Enteropathy*

\begin{tabular}{lc}
\hline MHC marker & Relative risk \\
\hline HLA-A1 & 1.7 \\
HLA-A29 & 7.0 \\
HLA-B8 & 17.8 \\
HLA-DR3 & 15.9 \\
SC01 & 11.4 \\
[HLA-B8, DR3, SC01] & 12.9 \\
HLA-B44 & 2.3 \\
HLA-DR7 & 3.7 \\
FC31 & 2.6 \\
BF*F & 2.0 \\
[HLA-B44, DR7, FC31] & 13.4 \\
HLA-B7 & 0.3 \\
HLA-DR1 & $<0.2$ \\
HLA-DR2 & 0.1 \\
HLA-DR4 & 0.2 \\
HLA-DR5 & 0.8 \\
HLA-DRw6 & 0.4 \\
\hline
\end{tabular}

* Relative risk $=$ (no. of patients with marker $\times$ no. of controls without marker)/(no. of patients without marker $\times$ no. of controls with marker). In this calculation, homozygotes and heterozygotes each count as 1 . 
Table IV. Distribution and Odds Ratios of HLA-DR3 and DR7 Homozygotes and Heterozygotes among Patients with Gluten-sensitive Enteropathy*

\begin{tabular}{|c|c|c|c|c|c|c|c|}
\hline & \multicolumn{2}{|l|}{ Homozygous } & \multicolumn{3}{|l|}{ Heterozygous } & \multirow[b]{2}{*}{ Other: $X 0$ or $X X$} & \multirow[b]{2}{*}{$0 / 0$} \\
\hline & HLA-DR $3 / 3$ or $3 / 0$ & HLA-DR $7 / 7$ or $7 / 0$ & HLA-DR3/DR7 & HLA-DR3/X & $7 / X$ & & \\
\hline Expected & 5.03 & 2.63 & 6.42 & 4.57 & 2.92 & 1.50 & 0.04 \\
\hline Observed & 4 & 1 & 10 & 5 & 3 & 1 & 0 \\
\hline$\chi^{2}$ & 0.21 & 1.01 & 2.00 & 0.04 & 0.002 & 0.17 & - \\
\hline Odds ratio & 56.5 & 70.7 & 235.6 & & & & \\
\hline
\end{tabular}

* DRX is an HLA-DR specificity other than DR3 or DR7. DR0 is HLA-DR blank or failure to react with typing antisera.

markers, it was of interest to observe the distribution of homozygotes and heterozygotes for HLA-DR3 and DR7. Both by the odds ratio calculation and Rotter's analysis (29) for the number of heterozygotes exceeding the sum of the number of homozygotes, there was an increase in heterozygotes. However, this increase was not statistically significant and appeared to consist of a paucity of homozygotes as well as an increase in heterozygotes. The approximate fit of the distribution to the Hardy-Weinberg equilibrium of homozygotes for HLA-DR3 and DR7 and DR3/DR7 heterozygotes in patients with glutensensitive enteropathy suggests that susceptibility is inherited primarily as a recessive trait. This is also true of type I diabetes mellitus if one analyzes for $B F^{*} F 1$ using the Thomson-Bodmer method $(30,31)$. Although families with more than one affected sib with gluten-sensitive enteropathy are rare, they tend to be HLA-identical, in keeping with largely recessive inheritance $(32,33)$.

In type 1 diabetes mellitus, the increase in HLA-DR3 is the result solely of an increase in DR3-bearing extended haplotypes, whereas the increase in HLA-DR4 is associated with an increase both in specific DR4-bearing extended haplotypes and an increase in DR4 haplotypes that are not recognizably extended. The situation in gluten-sensitive enteropathy is closely analogous with the increase in HLA-DR3 consisting only of one extended haplotype, whereas the increase in HLA-DR7 is in both one extended haplotype and in nonextended DR7. In both diseases, it may be that the nonextended haplotypes associated with HLADR4 and DR7, respectively, contain fragments of extended haplotypes.

The extended haplotype [HLA-B8, DR3, SC01] appears to mark a number of diseases, including gluten-sensitive enteropathy, as demonstrated here. It is important to know whether alleles at other loci on the haplotype than those examined are the same or different in patients with different diseases having the same marker haplotype. Clues to the answer to this question are provided by the differences in GLO and HLA-A allele distribution between [HLA-B8, DR3, SC01] in diabetes and gluten-sensitive enteropathy. In the former disease, it is primarily the GLO2marked haplotype that is increased, whereas in gluten-sensitive enteropathy it is both GLO1- and GLO2-marked [HLA-B8, DR3, SC01]. In gluten-sensitive enteropathy, most [HLA-B8, DR3, SC01] do not bear HLA-A1, but have a variety of other alleles; in diabetes, they are mostly HLA-A 1 . This suggests that [HLA-B8, DR3, SC01]-bearing chromosomes that carry type I diabetes susceptibility may differ from those that carry glutensensitive enteropathy, although they obviously have had a common ancestor and some chromosomes could carry both suscep- tibility genes. The variability in HLA-A and GLO alleles on gluten-sensitive enteropathy patient [HLA-B8, DR3, SC01] suggests that the MHC-linked susceptibility locus for gluten-sensitive enteropathy is probably closer to the telomere of chromosome 6p than $G L O$ and closer to the centromere than $H L A-A$. A similar concept, but without the direct evidence from study of haplotypes, is embodied in using the population relative risks of HLA-A1, B8, and DR3 to identify the "primary" marker for gluten-sensitive enteropathy (34).

Both the limitation and danger of comparing relative risk values too precisely for susceptibility gene localization or other purposes are clearly seen in Table III. Although the relative risk for the complete extended haplotype [HLA-B44, DR7, FC31] exceeds the relative risks of its component alleles by severalfold, this is not true of [HLA-B8, DR3, SC01], reflecting the fact that on both normal and patient chromosomes a large part of HLAB8, DR3, and SC01 occurs as the extended haplotype. This is less true of the components of [HLA-B44, DR7, FC31]. The relative risk for any marker, whether it is a single allele such as $B F^{*} F 1$ in type I diabetes or an extended haplotype such as [HLAB44, DR7, FC31] in gluten-sensitive enteropathy, is greatly influenced by its frequency in the normal population. In general, the rarer the marker in the control population, the greater its tendency to overaffect the relative risk. If one uses relative risks too literally, one could conclude that since HLA-B8 has a higher relative risk than either HLA-A1 or HLA-DR3, the gluten-sensitive enteropathy susceptibility gene must be the same as HLAB8 or close to $H L A-B$. This contradicts the conclusion that HLADR7 or HLA-DR is primary as judged from the fact that HLADR7 has a higher relative risk than either HLA-B44 or FC31. It is certainly not consistent with HLA-A29 being primary because it has the highest relative risk on the haplotype HLA-A29, B44, DR7, FC31.

The fact that the majority of patient [HLA-B8, DR3, SC01] do not carry HLA-A1 accounts mainly for the lower relative risk of HLA-A1 compared with that of HLA-B8 or DR3 observed in our study. In addition, there certainly is some population heterogeneity of allele frequencies in published studies, as judged from HLA studies of Caucasian patients in different regions (14). Our HLA-A1 relative risk of 1.7 is somewhat lower than that reported in a U.S. population at 3.64 (35) but is comparable to that observed in an African Caucasian group at 1.91 (36) and a Western European population at 1.68 (32).

Our findings strongly suggest that HLA-DR3 or DR7 themselves, as currently defined, are not susceptibility genes for glutensensitive enteropathy. Rather, they are markers for such genes on extended haplotypes. 


\section{Acknowledgments}

We thank Deborah Marcus, Catherine Ramaika, Carroll Goldsmith, and Sharon Martin Alosco for their expert technical assistance and Ada Watson for coordinating some of the family studies.

This work was supported by National Institutes of Health grants AM-26844, HD-17461, CA-20531, CA-06516, and HL-29583 and a grant from the American Red Cross.

\section{References}

1. Falchuk, Z. M., and W. Strober. 1972. HL-A antigens and adult coeliac disease. Lancet. ii: 1310.

2. Stokes, P. L., P. Asquith, G. K. T. Holmes, P. Mackintosh, and W. T. Cooke. 1973. Inheritance and influence of histocompatibility (HLA) antigens in adult coeliac disease. Gut. 14:627-630.

3. Keuning, J. J., A. S. Pena, A. Van Leeuwen, J. P. Van Hooff, and J. J. Van Rood. 1976. HLA-DW3 associated with coeliac disease. Lancet. i:506-507.

4. Demarchi, M., I. Borelli, E. Olivetti, P. Richiardi, P. Wright, N. Ansaldi, C. Barbera, and B. Santini. 1979. Two HLA-D alleles are associated with coeliac disease. Tissue Antigens. 14:309-316.

5. Singal, D. P., and M. A. Blajchman. 1973. Histocompatibility (HL-A) antigens, lymphocytotoxic antibodies and tissue antibodies in patients with diabetes mellitus. Diabetes. 22:429-432.

6. Nerup, J., P. Platz, D. Ortved Andersen, M. Christy, J. Lyngsoe, J. E. Poulsen, L. P. Ryder, L. Staub-Nielsen, M. Thomsen, and A. Svejgaard. 1974. HL-A antigens and diabetes mellitus. Lancet. ii:864866.

7. Cudworth, A. G., and J. C. Woodrow. 1976. Genetic susceptibility in diabetes mellitus: analysis of the HLA association. Br. Med. J. 2:846848.

8. Tiwari, L., and P. I. Terasaki. 1985. Juvenile diabetes mellitus (insulin-dependent). In HLA and Disease Associations. Springer-Verlag New York Inc., New York. 185-210.

9. Raum, D., C. A. Alper, R. Stein, and K. H. Gabbay. 1979. Genetic marker for insulin-dependent diabetes mellitus. Lancet. i:1208-1210.

10. McCann, V. J., J. McCluskey, P. H. Kay, P. J. Zillio, F. T. Christiansen, and R. L. Dawkins. 1983. HLA and complement genetic markers in diabetic retinopathy. Diabetologia. 24:221.

11. Raum, D., Z. Awdeh, E. J. Yunis, C. A. Alper, and K. H. Gabbay. 1984. Extended major histocompatability complex haplotypes in type I diabetes mellitus. J. Clin. Invest. 74:449-454.

12. Awdeh, Z. L., and C. A. Alper. 1980. Inherited structural polymorphism of the fourth component of human complement (C4). Proc. Natl. Acad. Sci. USA. 77:3576-3580.

13. Awdeh, Z. L., D. Raum, and C. A. Alper. 1979. Genetic polymorphism of the fourth component of human complement: detection of heterozygotes. Nature (Lond.). 282:205-207.

14. Mauff, G., C. A. Alper, Z. Awdeh, J. R. Batchelor, J. Bertrams, G. Bruun-Petersen, R. L. Dawkins, P. Démant, J. Edwards, H. GrosseWilde, G. Hauptmann, P. Klouda, L. Lamm, E. Mollenhauer, C. Nerl, B. Olaisen, G. O'Neill, C. Rittner, M. H. Roos, V. Skanes, P. Teisberg, and L. Wells. 1983. Statement on the nomenclature of human C4 allotypes. Immunobiology. 164:184-191.

15. Alper, C. A., T. Boenisch, and L. Watson. 1972. Genetic polymorphism in human glycine-rich beta-glycoprotein. J. Exp. Med. 135: 68-80.

16. Alper, C. A. 1976. Inherited structural polymorphism in human C2: evidence for genetic linkage between $C 2$ and Bf. J. Exp. Med. 144: 1111-1115.

17. Bodmer, W. F., J. R. Batchelor, J. G. Bodmer, H. Festenstein, and P. J. Morris, editors. 1978. Histocompatibility Testing 1977. Munksgaard, Copenhagen, 612.

18. Kömpf, J., S. Bissbort, S. Gussmann, and H. Ritter. 1975. Poly- morphism of red cell glyoxalase I (E.C.4.4.1.5). A new genetic marker in man. Humangenetik. 27:141-143.

19. Alper, C. A., D. Raum, S. Karp, Z. L. Awdeh, and E. J. Yunis. 1983. Serum complement 'supergenes' of the major histocompatibility complex in man (complotypes). Vox Sang. 45:62-67.

20. Awdeh, Z. L., D. Raum, E. J. Yunis, and C. A. Alper. 1983. Extended HLA-complement-glyoxalase allele haplotypes: evidence for T/t-like complex in man. Proc. Natl. Acad. Sci. USA. 80:259-263.

21. Alper, C. A., Z. L. Awdeh, D. D. Raum, and E. J. Yunis. 1982. Extended major histocompatibility complex haplotypes in man: role of alleles analogous to murine t mutants. Clin. Immunol. Immunopathol. 24:276-285.

22. Nelson, D. L., Z. M. Falchuk, D. Kasarda, and W. Strober. 1975. Gluten-sensitive enteropathy: correlation of organ culture behavior with HL-A status. Clin. Res. 23:254A. (Abstr.)

23. Falchuk, Z. M., D. L. Nelson, A. J. Katz, J. E. Bernardin, D. D. Kasarda, N. E. Hague, and W. Strober. 1980. Gluten sensitive enteropathy: influence of histocompatibility type on gluten sensitivity in vitro. J. Clin. Invest. 66:227-233.

24. Fleischnick, E., Z. L. Awdeh, D. Raum, J. Granados, S. M. Alosco, J. F. Crigler, Jr., P. S. Gerald, C. M. Giles, E. J. Yunis, and C. A. Alper. 1983. Extended MHC haplotypes in 21-hydroxylase-deficiency congenital adrenal hyperplasia: shared genotypes in unrelated patients. Lancet. i: 1269-1275.

25. Dupont, B., S. E. Oberfeld, E. M. Smithwick, T. D. Lee, and L. S. Levine. 1977. Close genetic linkage between HLA and congenital adrenal hyperplasia (21-hydroxylase deficiency). Lancet. ii:1309-1311.

26. White, P. C., D. D. Chaplin, J. H. Weis, B. Dupont, M. I. New, and J. G. Seidman. 1984. Two steroid 21-hydroxylase genes are located in the murine $\mathrm{S}$ region. Nature (Lond.). 312:465-470.

27. Carroll, M. C., R. D. Campbell, and R. R. Porter. 1985. The mapping of 21-hydroxylase genes adjacent to complement component $\mathrm{C} 4$ genes in HLA, the major histocompatibility complex. Proc. Natl. Acad. Sci. USA. 82:521-525.

28. Svejgaard, A., and L. P. Ryder. 1981. HLA genotype distribution and genetic models of insulin-dependent diabetes. Ann. Hum. Genet. 45:293-298.

29. Rotter, J. I., C. E. Anderson, R. Rubin, J. E. Congleton, P. I. Terasaki, and D. L. Rimoin. 1983. HLA genotypic study of insulindependent diabetes: the excess of DR3/DR4 heterozygotes allows rejection of the recessive hypothesis. Diabetes. 32:169-174.

30. Raum, D., Z. Awdeh, and C. A. Alper. 1981. BF types and the mode of inheritance of insulin-dependent diabetes mellitus. Immunogenetics. 12:59-74.

31. Morton, N. E., A. Green, T. Dunsworth, A. Svejgaard, J. Barbosa, S. S. Rich, L. Iselius, P. Platz, and L. P. Ryder. 1983. Heterozygous expression of insulin-dependent diabetes mellitus (IDDM) determinants in the HLA system. Am. J. Hum. Genet. 35:201-213.

32. Betuel, H., L. Gebuhrer, L. Descos, J. Bertrand, F. Freycon, and J. C. Lepetit. 1981. Celiac disease and its association with HLA markers. In Histocompatibility Testing 1980. P. I. Terasaki, editor. UCLA Tissue Typing Laboratory, Los Angeles. 668-672.

33. Greenberg, D. A., S. E. Hodge, and J. I. Rotter. 1982. Evidence for recessive and against dominant inheritance at the HLA-"linked" locus in coeliac disease. Am. J. Hum. Genet. 34:263-277.

34. Tiwari, J. L., and P. I. Terasaki. 1985. Celiac disease. In HLA and Disease Associations. Springer-Verlag New York Inc., New York. 236-242.

35. Falchuk, Z. M., G. N. Rogentine, and W. Strober. 1972. Predominance of histocompatibility antigen HL-A8 in patients with glutensensitive enteropathy. J. Clin. Invest. 51:1602-1605.

36. Mougenot, J. F., J. Hors, M. Schmid, M. Cathelineau, J. Navarro, and C. Polonovski. 1977. Les antigenes HLA dans la maladie coeliaque et l'intolerance digestive aux proteines du lait de vache chez l'enfant. Gastroenterol. Clin. Biol. 1:507-514. 